\title{
BMJ Open Prescriber preference for a particular tumour necrosis factor antagonist drug and treatment discontinuation: population-based cohort
}

\author{
Anat Fisher, ${ }^{1}$ Ken Bassett, ${ }^{1,2}$ James M Wright, ${ }^{1,3}$ M Alan Brookhart,, \\ Hugh J Freeman, ${ }^{3}$ Colin R Dormuth ${ }^{1}$
}

To cite: Fisher A, Bassett $\mathrm{K}$, Wright JM, et al. Prescriber preference for a particular tumour necrosis factor antagonist drug and treatment discontinuation: population-based cohort. BMJ Open 2014;4:e005532. doi:10.1136/bmjopen-2014005532

- Prepublication history for this paper is available online. To view these files please visit the journal online (http://dx.doi.org/10.1136/ bmjopen-2014-005532).

Received 23 April 2014 Revised 10 September 2014 Accepted 11 September 2014

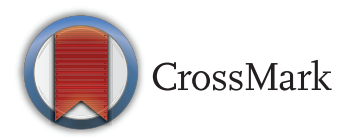

For numbered affiliations see end of article.

Correspondence to Dr Anat Fisher; anat.fisher@ti.ubc.ca

\section{ABSTRACT}

Objective: To assess the effect of physician preference for a particular tumour necrosis factor $\alpha$ (TNF) antagonist on the risk of treatment discontinuation in rheumatoid arthritis.

Design: Population-based cohort study.

Setting: British Columbia administrative health data (inpatients, outpatients and pharmacy).

Participants: 2742 British Columbia residents who initiated a first course of a TNF antagonist between 2001 and December 2008, had been diagnosed with rheumatoid arthritis, and were treated by 1 of 58 medium-volume to high-volume prescribers.

Independent variable: A level of physician preference for the drug (higher or lower) was assigned based on preceding prescribing records of the care-providing physician. Higher preference was defined as at least $60 \%$ of TNF antagonist courses initiated in the preceding year. Sensitivity analysis was conducted with different thresholds for higher preference.

Main outcome measure: Drug discontinuation was defined as a drug-free interval of 180 days or switching to another TNF antagonist, anakinra, rituximab or abatacept. The risk of discontinuation was compared between different levels of physician preference using survival analysis.

Results: Higher preference for the prescribed TNF antagonist was associated with improved persistence with the drug (4.28 years $(95 \% \mathrm{Cl} 3.70$ to 4.90$)$ vs 3.27 (2.84 to 3.84), with log rank test $p$ value of 0.017). The adjusted HR for discontinuation was significantly lower in courses of drugs with higher preference $(0.85(0.76$ to 0.96$))$. The results were robust in a sensitivity analysis.

Conclusions: Higher physician preference was associated with decreased risk of discontinuing TNF antagonists in patients with rheumatoid arthritis. This finding suggests that physicians who strongly prefer a specific treatment help their patients to stay on treatment for a longer duration. Similar research on other treatments is warranted.

\section{Strengths and limitations of this study}

First study to explore within-physician variation in prescribing habits, specifically the effect of prescriber preference to a drug on the decision to discontinue the drug.

- The universal nature of the Canadian healthcare system and a systematic and standardised approach to data collection in British Columbia, which ensured the generalisability of our results, as well as the large sample and prolonged follow-up.

- To conquer the absence of access to clinical data, we used multiple proxy variables to adjust for disease severity.

- Physician preference was not directly measured but instead based on previous prescribing habits.

\section{INTRODUCTION}

The term 'physician preference' usually refers to favouring a particular drug or a therapeutic group among several alternatives, and it has been shown to predict treatment choice. ${ }^{1-4}$ In studies of administrative health (claim) data, this preference is often determined by identifying dispensing of drug prescribed by the specific physician in a predetermined period, prior to the event of interest (a new prescribing). Despite an association with new prescribing decisions, the role of physician preference in treatment discontinuation has not been studied. Recently, the term 'preference' has also been used to describe a second phenomenon-in the context of treatment discontinuation, it was used to describe the baseline risk of discontinuing treatment in patients treated by a specific physician (the physician 'preference for discontinuation'). ${ }^{5}$ This baseline risk may 
differ among physicians because physicians may respond differently to similar clinical situations such as decreased benefit or harmful events. They could recommend patients to discontinue treatment (with or without switching to a second drug) or to persist with the treatment (but to adjust dose, add-on a second drug or be under frequent watch). In this paper, we use the term 'preference' to describe the first phenomenon (physician's favourite drug) and 'physician-specific discontinuation risk' to describe the second.

Treatment with tumour necrosis factor $\alpha$ (TNF) antagonists in patients with rheumatoid arthritis (RA) was considered especially sensitive to physician preference for two main reasons. First, during the study period (2001-2009) there was limited clinical evidence on the comparative effectiveness of the drugs, mainly due to the absence of head-to-head randomised clinical trials, but also because participants in placebo-controlled trials were not representative of patients treated in routine clinical settings. ${ }^{6-9}$ Second, published indications for discontinuation of TNF antagonists were vague and confusing, and therefore care-providing physicians could reasonably be expected to reach different clinical decisions given the same clinical situation. Consequently, the decisions about which TNF antagonist to prescribe first and when to discontinue treatment were likely subject to physicians' individual preference.

This study analysed data of first courses of a TNF antagonist in British Columbia patients with RA. The prescriber recorded on the first dispensing claim for a TNF antagonist was used as a proxy of the care-providing physician. The study objective was to estimate the effect of physician preference on the risk of discontinuation. The null hypothesis tested was that physician preference for a TNF antagonist when treatment has been initiated does not influence the risk of discontinuing the treatment in patients with RA.

\section{PATIENTS AND METHODS}

The study cohort was identified using four British Columbia Ministry of Health administrative databases: PharmaNet (prescription dispensing data), Medical Service Plan (MSP) registration information (demographic data), MSP Payment Information (fee-for-service payments to physicians and alternative providers), and the Discharge Abstract Database (hospital separations). The databases were linkable using a de-identified patient and physician numbers. Follow-up data were available from 1995 until 31 December 2009. The study cohort included British Columbia residents who fulfilled all of three conditions: (1) first exposure to a TNF antagonist between 1 March 2001 and 31 December 2008; (2) a diagnosis of RA and (3) TNF antagonist treatment initiated by a medium-volume or high-volume prescriber, defined as a prescriber who initiated five or more courses of TNF antagonists in patients with RA during the years 2001-2008. Exposure to a TNF antagonist was based on one or more recorded dispensing claims for infliximab, adalimumab or etanercept between March 2001 and 31 December 2008. We excluded patients who were previously treated with anakinra, rituximab or abatacept to decrease heterogeneity in the population analysed. The first dispensing claim for a TNF antagonist was used to identify the study drug (infliximab, adalimumab or etanercept), index date and prescriber for each patient in the cohort. We defined a prestudy period of 3 years preceding the index date during which patients were required to have continuous provincial medical service coverage. A gap shorter than 30 days was not considered to be an interruption in coverage. The prestudy period ensured a standard run-in period of at least 3 years without TNF antagonist exposure and a standard period during which diagnosis of RA was identified. Patients with RA were selected based on similar criteria to previous studies in British Columbia. ${ }^{10-12}$ Either two outpatient visits in physician clinics with a diagnosis code of RA (International Classification of diseases-9 714) at least 60 days apart, or one hospitalisation with recorded discharge diagnosis of RA was required. Patients were excluded if sex or date of birth was not available, if they had a concurrent diagnosis of Crohn's disease (based on at least one outpatient or inpatient diagnosis code in the prestudy period) or if they were younger than 18 years at the index date (to remove patients with juvenile RA). We also excluded patients initiated on TNF antagonist treatment by prescribers who cumulatively initiated less than five patients in the RA cohort (low-volume prescribers).

Physician preference was determined for each patient at the index date and coded as a Bernoulli variable. It reflects prescribing patterns of the individual physician (who started this course) during the preceding year (figure 1). For each individual patient, we determined the date of course initiation, which the prescriber recorded in the first dispensing event and the drug (infliximab, adalimumab or etanercept). From first dispensing records for each patient in the study cohort, we identified other patients that received any TNF antagonists from the same prescriber during the year preceding the index date of the current patient, and the drug they were treated with. Next we calculated the proportion of these patients who received the same drug as the patient of interest. Based on this proportion, we categorised the prescriber preference to 'higher' when the calculated proportion for this drug and that prescriber was $60 \%$ or higher, and 'lower' otherwise. We expected that this threshold would provide reasonable assurance that a course categorised as having 'higher' preference for drug actually reflects that the prescriber favoured that particular TNF antagonist at this time, even if only two TNF antagonists were available. We reassessed preference for drug based on annual data to allow for changes in preference over time and for accommodating the recent availability of adalimumab. Adalimumab was available in Canada since October 2004, while infliximab and etanercept were on the market since 2001. By 


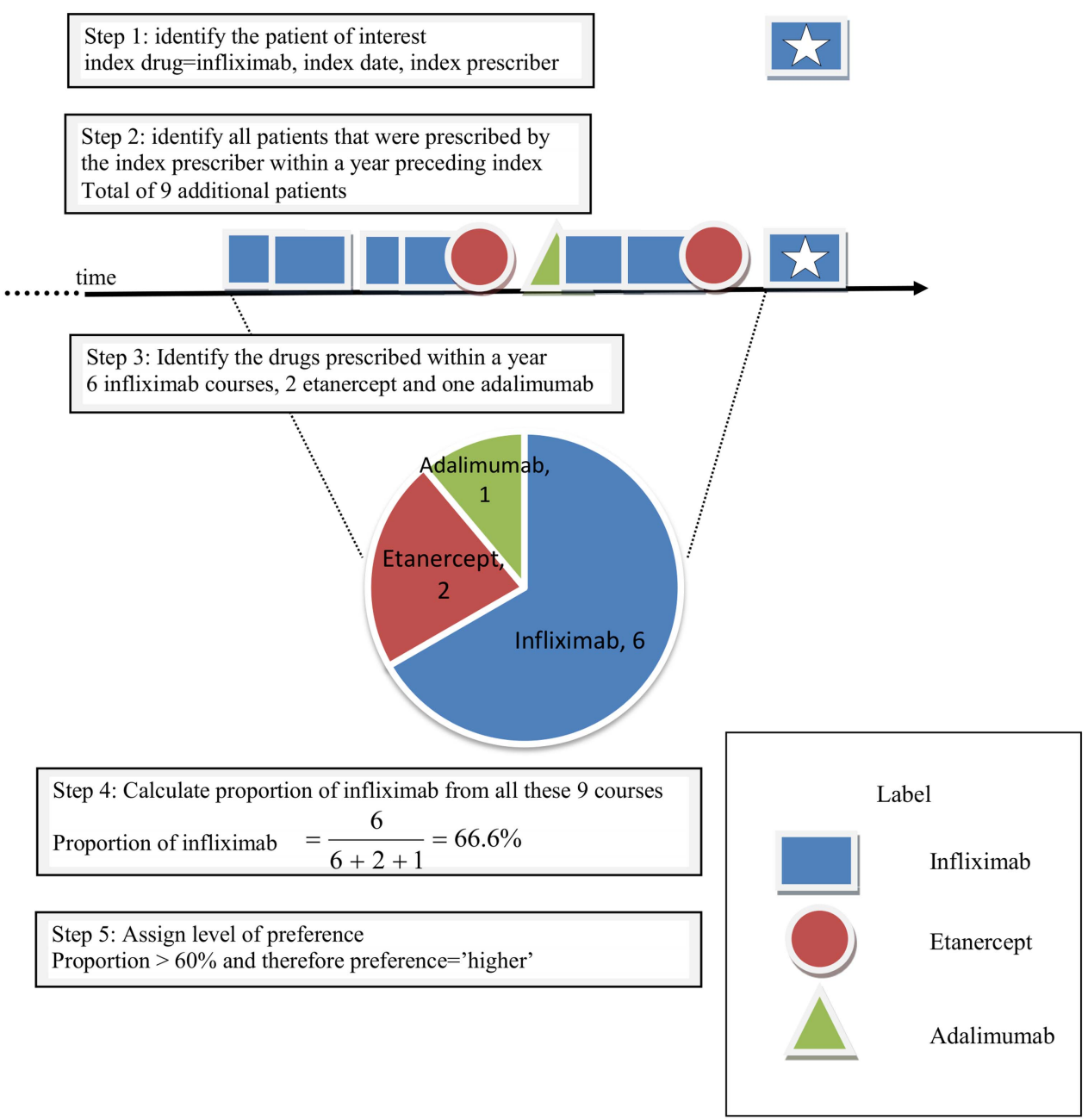

Figure 1 Calculating the value of the independent variable physician preference.

including a year of data, differently from other studies that used only the last prescription in assigning preference, ${ }^{1-4}$ we created a more accurate indicator. This way we minimised the effect of factors not related to preference that might have influenced a specific prescribing decision. Sensitivity analysis was conducted to examine the robustness of main results, using thresholds of $70 \%$ or $80 \%$ for the level of physician preference.

Multiple covariates, mainly patient characteristics that may influence drug selection and/or discontinuation were included in the final models and are listed in table 1 . We were limited by lack of access to clinical data and inability to adjust for clinical variables not captured in administrative health data, such as disease severity. Hence, we used multiple proxies, including disease duration, age, extra-articular involvement, antirheumatic drug use, health services utilisation and consumption of pain medications to adjust for disease severity. In order to control for secular trends and late availability of adalimumab in Canada, we included a categorical variable for the year of treatment initiation.

The outcome variable was discontinuation defined as either switching to another TNF antagonist including certolizumab and golimumab, anakinra, rituximab or abatacept ('biologic' antirheumatic drugs), or a drug-free interval of 180 days after exhaustion of the dispensed days-supply of the latest refill. Discontinuation date was set to the end of the days-supply of the last refill before the 180-day drug-free interval or the date of the first dispensing of a second 'biologic' antirheumatic drug, whichever was earliest. Unless discontinuation occurred, patients were followed up until either 31 December 2009 (end of follow-up period) or an interruption of more than 6 days in the provincial MSP coverage, at which point data were considered censored. The most common causes of coverage interruptions were death and emigration from the province. Change of dose of the TNF antagonist or addition of a second drug (not 'biologic' antirheumatic drug) was not considered discontinuation.

Discrepancies between recorded days-supply and dispensed quantity were common in the PharmaNet database; hence, we also calculated days-supply based on the quantity dispensed, which, if required, was imputed using the recorded total cost. Cost was considered the most accurate and reliable field, since this field serves for claim and payment processing. We used the longest 
Table 1 List of covariates included in the final models

Variable
Demographics
Age at index
The annual deductible for prescription cost
at index (Fair PharmaCare)
Geographical area at index
Clinical status
Physicians encounters in the year
preceding the index date
Number of inpatient admissions in the year
preceding the index date
Comorbidities (presence and severity)
during the prestudy period

\section{Disease duration}

The presence of extra-articular manifestation during the prestudy period

\section{Drug therapies \\ Concomitant MTX during 200 days \\ preceding the index date}

Dispensing claims for NSAIDs during the year preceding the index date

Number of different antirheumatic drugs dispensed in the prestudy period

\section{Other}

Calendar year at index date

\section{Description}

\section{Bernoulli variable}

Four categories: 18-29, 30-69, 70-79 and $\geq 80$ years (categories were assigned based on similar discontinuation risk in preliminary analysis, which was not linear)

The deductible is based on annual income. ${ }^{13}$ Six categories: annual deductible of $\$ 0, \$ 1-\$ 500, \$ 501-\$ 2250$, > $\$ 2250$, other programmes and no coverage

Based on first 3 digit of postal code. Five mutually exclusive categories: Greater Vancouver, Greater Victoria, Vancouver Island, urban areas and rural areas

\section{Continuous variable}

Four categories: $0,1,2$ and $>2$

Charlson comorbidity score ${ }^{14}$ was determined using Quan's algorithm for administrative databases, ${ }^{15}$ excluding rheumatic diseases. At least two outpatient or one inpatient encounter with the diagnosis were required. Four categories: 0, 1, 2 and $>2$

Measured from the first diagnosis of RA (inpatient or outpatient) in the data. RA disease duration captured in Canadian administrative data has been found to agree with reported duration by physicians. ${ }^{16}$ Continuous variable Based on recorded at least one diagnoses with ICD-9 codes 3571, 3596, $7141,71481,7142$ (outpatient) or ICD-10 codes G636, G737, I39, 1418, J990, M050, M051, M052, M053 (inpatient). Bernoulli variable

Based on mean plus two SDs of between-dispensing intervals of MTX in the study cohort. Bernoulli variable

Bernoulli variable

Dispensing claims of 10 drugs were included: MTX, hydroxychloroquine, sulfasalazine, leflunomide, azathioprine, minocycline, penicillamine, sodium aurothiomalate, prednisone, and intra-articular triamanolone or methylprednisolone. Four categories: no drug, 1-2, 3-6 and >6 different drugs

The variable allowed controlling for secular trends in clinical practice ${ }^{17-20}$ and availability of drugs. Eight yearly categories were included for the years 2001-2008

ICD-10, International Classification of Diseases, 10th edition; MTX, methotrexate; NSAID, non-steroidal anti-inflammatory drug; RA, rheumatoid arthritis.

duration of days-supply, recorded or calculated, to determine both the length of drug-free intervals and a discontinuation date.

\section{Sample size calculation}

Assuming 1:1 ratio of courses with 'higher' preference versus courses with 'lower' preference and in the absence of prior estimates, if the true HR for discontinuing courses with 'higher' preference relative to courses with 'lower' preference is 0.8 , we required 847 courses at each preference level to be able to reject the null hypothesis with type I error probability of 0.05 and power of 0.80 .

\section{Statistical analysis}

Patients' characteristics were compared across the three drugs. The adjusted risk of discontinuation was compared using two different approaches in a Cox proportional hazards regression. First, we conducted an analysis of non-clustered data. The multivariate model also included a series of Bernoulli variables, one for each physician ( $1=$ the particular physician; $0=$ other physicians), which allowed individualisation of the risk of drug discontinuation by physician (physician-specific discontinuation risk). In the second approach, a marginal model of clustered data allowed an adjustment for possible correlation between patients treated by the same physician. ${ }^{21-23}$ We tested for model assumptions 
Figure 2 Participants' flow (RA, rheumatoid arthritis; TNF, tumour necrosis factor $\alpha$ ).
7212 patients treated with TNF antagonists until December 2009 in the province of British Columbia (BC)

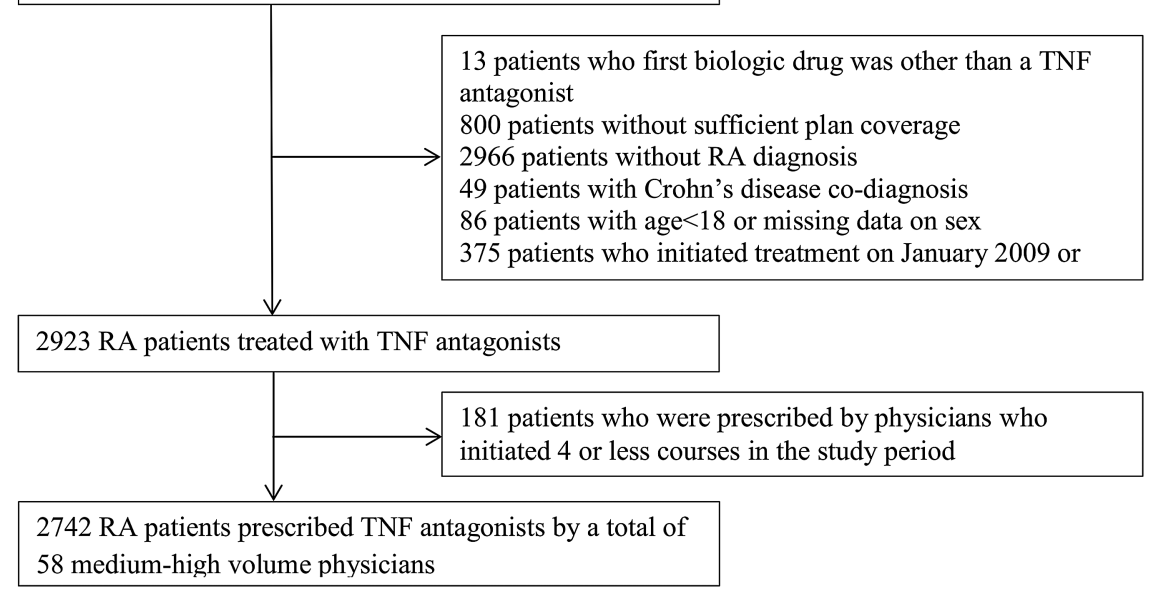

(proportional hazard and absence of interactions) and found them to be valid. We also checked for the linearity of continuous variables and categorised non-linear variables. All statistical tests were two sided. All calculations were performed using the SAS software package (SAS Institute Inc, Cary, North Carolina, USA).

\section{RESULTS}

The study cohort included 2742 patients with RA prescribed by a total of 58 medium-high volume physicians.
Figure 2 presents reasons for excluding patients who used TNF antagonists from the analysis. Baseline characteristics across users of the three drugs are presented in table 2. Not only was etanercept the most frequently prescribed drug (1718 patients, 63\%), but it was also the only drug prescribed by all 58 study physicians. Etanercept was usually initiated by a physician with high preference for etanercept (70\% of etanercept courses). Infliximab or adalimumab, however, were usually initiated by physicians with lower preference for these

Table 2 Baseline characteristics

\begin{tabular}{|c|c|c|c|c|}
\hline Variable & Infliximab & Adalimumab & Etanercept & $\begin{array}{l}\text { p Value (between- } \\
\text { group differences) }\end{array}$ \\
\hline Number of patients (\% from cohort) & $571(21)$ & $453(16)$ & $1718(63)$ & \\
\hline $\begin{array}{l}\text { Number of prescribers (based on first dispensing } \\
\text { event for each patient; total } 58 \text { prescribers) }\end{array}$ & 49 & 46 & 58 & \\
\hline $\begin{array}{l}\text { Patients treated with a drug with higher preference, } \\
\mathrm{N}(\%)\end{array}$ & $193(34)$ & $84(19)$ & $1198(70)$ & $<0.0001$ \\
\hline \multicolumn{5}{|l|}{ Demographics } \\
\hline Females, N (\%) & $403(71)$ & $326(72)$ & $1239(72)$ & 0.77 \\
\hline Age at index, median years (range) & $56(18-87)$ & $58(22-91)$ & $56(18-92)$ & 0.003 \\
\hline \multicolumn{5}{|l|}{ Annual deductible for prescription cost, N (\%) } \\
\hline Very low $(\$ 0)$ & $47(8.3)$ & $80(18)$ & $199(12)$ & $<0.0001$ \\
\hline Low $(\$ 1-\$ 500)$ & $33(5.8)$ & $53(12)$ & $152(8.9)$ & 0.004 \\
\hline Medium (\$501-\$2250) & $92(16)$ & $109(24)$ & $315(18)$ & 0.004 \\
\hline High $(>\$ 2250)$ & $35(6.1)$ & $38(8.4)$ & $143(8.3)$ & 0.22 \\
\hline Residence in Greater Vancouver/Victoria, N (\%) & $341(60)$ & $224(50)$ & $782(46)$ & $<0.0001$ \\
\hline \multicolumn{5}{|l|}{ Clinical status } \\
\hline Number of physician visits median (range) & $33(3-158)$ & $31(2-112)$ & $32(3-136)$ & 0.25 \\
\hline At least one admission to hospital, N (\%) & $104(18)$ & $63(14)$ & $340(20)$ & 0.01 \\
\hline Extra-articular manifestations, N (\%) & $28(4.9)$ & $14(3.1)$ & $60(3.5)$ & 0.23 \\
\hline Presence of comorbidity (score>0), N (\%) & $113(20)$ & $95(21)$ & $383(22)$ & 0.41 \\
\hline RA disease duration median, years (range) & $9.2(0.1-17.9)$ & $7.7(0.3-17.9)$ & $8.0(0-17.8)$ & 0.21 \\
\hline \multicolumn{5}{|l|}{ RA drugs } \\
\hline Concomitant MTX, N (\%) & $388(68)$ & $264(58)$ & $856(50)$ & $<0.0001$ \\
\hline Dispensing claims for NSAIDs, N (\%) & $307(54)$ & $214(47)$ & $923(54)$ & 0.04 \\
\hline $\begin{array}{l}\text { Number of different antirheumatic drugs, median } \\
\text { (range) }\end{array}$ & $4(0-8)$ & $4(0-8)$ & $4(0-9)$ & 0.46 \\
\hline
\end{tabular}




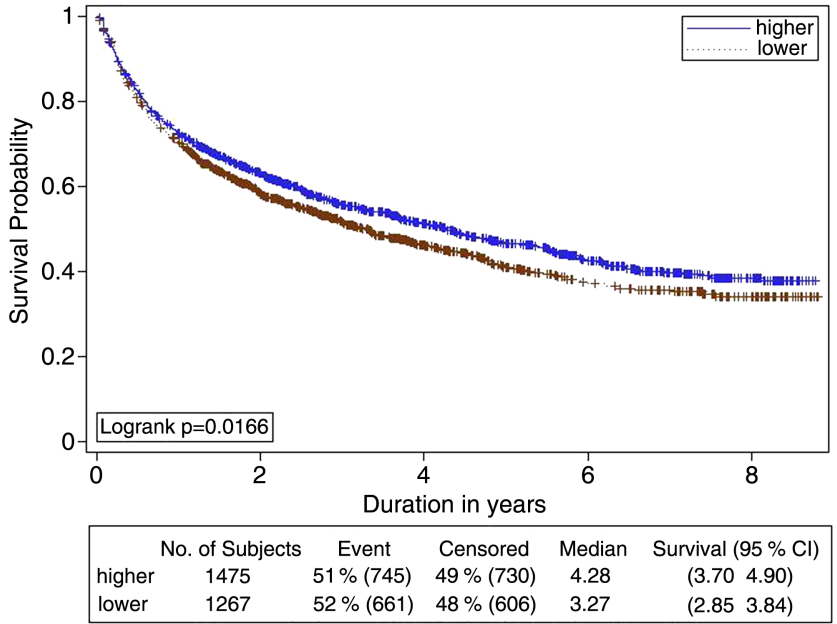

Figure 3 Persistence with tumour necrosis factor $\alpha$ antagonists by physician preference levels.

drugs (only $34 \%$ or $19 \%$ of courses were initiated by a physician with high preference for drug, respectively). Patients treated with adalimumab were significantly older and had a lower income (reflected in lower annual deductible level for prescribing costs). Patients treated with infliximab had the highest prevalence of concomitant methotrexate therapy and patients with etanercept the lowest. This reflects differences in the indications mentioned in the product monographs; while infliximab is indicated for use in combination with methotrexate, ${ }^{24}$ the monograph of etanercept mentioned that it "can be initiated in combination with methotrexate... or used alone". ${ }^{25}$ Similarly, while the provincial special authority policy requires that infliximab is used in combination with methotrexate (or other drug), such requirement does not exist for treatment with etanercept or adalimumab. ${ }^{26}$

Persistence with the three TNF antagonists was similar ( $\log$ rank test $\mathrm{p}$ value $=0.15)$. The product limit median persistence estimates were 3.9 years (95\% CI 3.0 to 5.3 ), 3.3 (2.6 to 4.0 ) and 3.9 years (3.4 to 4.4 ) for infliximab, adalimumab and etanercept, respectively. Higher physician preference was associated with improved persistence compared to lower preference (figure 3), with median time to discontinuation of 4.28 years (CI 3.70 to 4.90 ) in 1475 courses with 'high' preference, compared with 3.27 (2.85 to 3.84) in 1267 courses with 'low' preference. In both groups, about $50 \%$ of the patients discontinued and the other $50 \%$ were censored.

Higher physician preference was associated with a significant decrease of $14-15 \%$ in the adjusted hazard for discontinuation (table 3). No significant interaction between drug and preference was observed. The results of sensitivity analysis were similar, with overall adjusted HR between 0.85 and 0.88 in all models. The results of clustered data analyses with thresholds of $60 \%, 70 \%$ and $80 \%$ for physician preference were robust (table 3 ). The results of nonclustered data analyses for thresholds of $70 \%$ and $80 \%$, however, did not reach the significance level. That may be a result of decreased numbers of patients treated with a drug of higher physician preference and therefore decreased power to detect significant difference.

\section{DISCUSSION}

We demonstrated two types of variations of physician decisions to discontinue treatment with TNF antagonists: between-physician and within-physician. Between-physician variability is expressed as a different baseline risk of discontinuation depending on the treating physician in response to similar clinical situations, such as decreased benefit or a harmful event. We used the term physician-specific discontinuation risk for this phenomenon. It could be a result of differences in education or experience, adherence to different guidelines and possible differences in patient case mix. Within-physician variability, however, is a different response to a similar clinical situation (similar patients, similar drug effects) by the same physician. In this study we showed that this variability correlated to physician preference for drug-and specifically, higher physician preference for a specific TNF antagonist drug was associated with a decreased risk of discontinuing TNF antagonists in patients with RA. This preference most probably reflected the physician's beliefs regarding the relative effectiveness and safety of the specific drug compared to the alternatives,

Table 3 HRs for drug discontinuation, higher preference versus lower preference

\begin{tabular}{|c|c|c|c|}
\hline \multirow[b]{2}{*}{ Approach } & \multicolumn{3}{|l|}{ HR $(95 \% \mathrm{Cl})$} \\
\hline & $\begin{array}{l}\text { Preference threshold } \\
60 \%\end{array}$ & $\begin{array}{l}\text { Preference threshold } \\
70 \%\end{array}$ & $\begin{array}{l}\text { Preference threshold } \\
80 \%\end{array}$ \\
\hline Patients with higher preference, N (\%) & $1426(52)$ & $1234(45)$ & $987(36)$ \\
\hline \multicolumn{4}{|l|}{ Non-clustered data analysis } \\
\hline Crude & $0.88(0.79$ to 0.98$)$ & $0.89(0.80$ to 0.99$)$ & $0.83(0.81$ to 0.996$)$ \\
\hline Adjusted ${ }^{*}$ & $0.86(0.76$ to 0.98$)$ & $0.88(0.77$ to 1.01$)$ & $0.87(0.75$ to 1.004$)$ \\
\hline \multicolumn{4}{|l|}{ Marginal modelling of clustered data } \\
\hline Crude & $0.88(0.77$ to 1.001$)$ & $0.89(0.78$ to 1.01$)$ & $0.89(0.79$ to 1.01$)$ \\
\hline Adjusted $†$ & $0.85(0.76$ to 0.96$)$ & $0.87(0.78$ to 0.97$)$ & $0.87(0.78$ to 0.98$)$ \\
\hline
\end{tabular}


where a preferred drug is thought to be superior. When a physician prescribed a drug believed to be superior compared with comparators, patients were encouraged to stay longer on the drug and the risk of discontinuation, as measured in this study, decreased. We believe that a different response (level of encouragement) to a similar clinical situation applied in indefinite clinical situations, such as mild harmful effect or questionable benefit.

\section{Strengths and weaknesses of the study}

The main advantages of our study are the universal nature of the Canadian healthcare system and a systematic and standardised approach to data collection in British Columbia, which ensured the generalisability of our results, as well as the large sample and prolonged follow-up that increased the power of the study to detect differences in discontinuation risk. The main disadvantage is the absence of access to clinical data; hence adjustment to patient mix was difficult. To conquer this problem, we used multiple proxy variables to adjust for disease severity, such as extra-articular involvement, antirheumatic drug use, health services utilisation and consumption of pain medications.

There are several limitations to ascertaining physician preference based on previous prescribing habits. First, preference was not defined for all patients. For example, it was not defined for the first patient treated by the physician, or if the physician did not prescribe this class of drugs in the preceding year. Second, we assumed that increased loyalty for a particular TNF antagonist reflects preference, but we did not measure preference directly. Nevertheless, stated preference and prescribing habits were shown to correlate in previous research. ${ }^{27}$ Third, we measured preference at the time of treatment initiation and not at discontinuation, and preference might have changed over time. Finally, we assumed strong preference for one drug only and regarded situations in which the physician preferred two of the TNF antagonists to be a situation of treatment with a drug of lower preference.

\section{Overview of previous studies}

Treatment persistence was hypothesised to reflect therapeutic benefit and harm in chronic non-curable diseases, such as RA. ${ }^{28}$ In support of this hypothesis, previous studies in patients with RA using TNF antagonists demonstrated that the main reasons for discontinuing or switching were decreased benefit (36-67\% of the discontinuations) or perceived harm $(30-58 \%) .{ }^{29-34}$ As such, the risk of discontinuing treatment is assumed to be influenced solitarily by drug properties and patient characteristics. The demonstrated effect of care-provider characteristics, specifically prescribing habits, on the risk of discontinuation calls into question this hypothesis.

Between-physician differences in response to the same clinical situation (physician-specific discontinuation risk) have previously been studied in patients with RA treated by TNF antagonists. Differences in response to harm were reported by Cush ${ }^{1}$ based on an online survey of rheumatologists and in response to decreased benefit —by Zhang et $a l^{5}$ Using mixed effect model with a random intercept to cluster patients at physician level, Zhang demonstrated the importance of clustering by physicians, even after adjustment for baseline disease activity and improvement in disease activity.

Within-physician variation in prescribing decisions and the effect of physician preference have infrequently been studied. While the effect of physician preference on discontinuation has not been previously studied in this therapeutic class or in other conditions, physician preference was found to be an important predictor of drug selection in treatment initiation. Physician preference for therapeutic class was the most important determinant in initiating treatment with TNF antagonists compared with the alternative: prescribing synthetic antirheumatic drugs ${ }^{1}$ and in other treatment situations. ${ }^{34} 35$ Physician preference for an individual TNF antagonist was studied by Kamal et al. ${ }^{36}$ In response to mailed questionnaires, most American rheumatologists said they preferred etanercept over adalimumab or infliximab and considered etanercept the most efficacious of the three drugs with less harm. In our study, etanercept was the most prescribed TNF drug, and it was prescribed by all 58 medium-volume to high-volume prescribers.

\section{Explanations and interpretation}

We suggest several possible explanations for the finding of decreased risk of discontinuation with increased physician preference for the prescribed TNF antagonist. First, the results could be explained in light of the theory of cognitive dissonance. ${ }^{37}$ Dissonance is an uncomfortable feeling caused by holding conflicting ideas simultaneously. In line with this theory, treating with a drug believed to be inferior (lower preference) induces dissonance. In indefinite clinical situations, such as mild harmful effect or questionable benefit, early drug discontinuation supports the physician's belief that the selected drug was inferior compared to the alternatives. However, in a similar situation, a drug with a higher preference might be continued, to support a belief in its superiority. The interpretation of our results using the theory of cognitive dissonance is restricted by the lack of direct measurement of physician dissonance at the time of treatment discontinuation. The indirect measure of dissonance we used, physician preference, could also be influenced by factors unrelated to prescriber beliefs, such as limited availability of a drug or higher relative cost (although during the study period, availability or cost were not factors). Direct measures of cognitive dissonance are complicated and involve activities beyond the scope of the current study. ${ }^{38}$ Second, the observed association between higher preference and decreased risk of discontinuation might be confounded by experience with the drug, namely the total number of patients a physician has previously treated with the same drug. Increased experience with a specific drug 
may be associated with higher preference as a result of the algorithm we used to assign the level of preference, but also because of a tendency to continue doing what is familiar. Theoretically, increased experience with a specific drug would improve patient selection, and therefore is associated with improved benefit, decreased harm and decreased risk of discontinuation in these patients. Lastly, a physician's stated preference for a particular drug has been shown to correlate with patient preference. The selection of a specific TNF antagonist mostly depends on physician and/or patient preference because the benefit and harm profiles of the three drugs are considered to be similar ${ }^{39-41}$ despite limited relative effectiveness data. Physician and patient preferences for an individual TNF antagonist are likely to be correlated, as was shown in studies of non-steroidal antiinflammatory drug treatment in patients with RA. ${ }^{42}{ }^{43}$ In regard to TNF antagonists, studies showed that patients preferred shared treatment decisions or responsibility of the health professional when choosing a TNF antagonist. ${ }^{44}$ In addition, $67 \%$ of 77 Canadian rheumatologists surveyed reported concordance with the patient preference more than $80 \%$ of the time. ${ }^{45}$ If physician and patient preference for TNF antagonist do correlate, then improved persistence may have been a result of higher patient rather than physician preference.

Our findings suggest that administrative restrictions alone, without a change in physicians' preference and beliefs may not achieve the desired effect on practice. A change in drug coverage policy, for example, may achieve the desired effect in short term, since physicians would follow the new policy and prescribe mainly the reimbursed drug. In the longer term, however, unless a change in physician preference is achieved, the recommended drug would be discontinued early, and patients would be switched to a second-line drug. As a result, the overall effect of a change in policy alone would be less than desired. In a similar manner, the study indicates that the pharmaceutical industry may have a strong interest in influencing physicians despite administrative restrictions. ${ }^{46}$

\section{Unanswered questions and future research}

We theorise that in other conditions, when treating with a therapeutic class in which drugs are considered to have similar benefits and harms, a similar association exists. Further study of these conditions is required. In addition, we suggest exploring the explanation for the association observed, such as measuring cognitive dissonance during prescribing decision ${ }^{38}$ or patient preference. Awareness of the possible role of cognitive dissonance in clinical decision-making, based on beliefs not necessarily supported by clinical evidence, can contribute to the development of educational programmes for physicians. We also suggest exploring whether patients who had been initiated on a non-preferred TNF inhibitor were more likely to be switched to a preferred TNF antagonist. Further research is warranted to identify effective approaches to policy implementations.

\section{CONCLUSIONS}

Higher physician preference, estimated using their prescribing habits, was associated with decreased discontinuation risk in patients with RA treated with TNF antagonists. This finding highlights the limitation of introducing a new drug coverage policy without encouraging change in the physicians' drug preference. Similar research on other treatments is warranted.

\section{Author affiliations}

${ }^{1}$ Department of Anesthesiology, Pharmacology \& Therapeutics, University of British Columbia, Vancouver, British Columbia, Canada

${ }^{2}$ Department of Family Practice, University of British Columbia, Vancouver, British Columbia, Canada

${ }^{3}$ Department of Medicine, University of British Columbia, Vancouver, British Columbia, Canada

${ }^{4}$ Department of Epidemiology, Gillings School of Global Public Health, University of North Carolina, Chapel Hill, North Carolina, USA

Contributors AF, KB, JMW, MAB, HRF and CRD contributed to the study conception and design and interpretation of the data, drafting and revising the article critically for important intellectual content, and gave final approval of the version to be published. Analysis was performed by AF, as part of research conducted under the supervision of $\mathrm{CRD}$ as part of the requirements for her PhD project.

Funding The study was supported by the University of British Columbia Graduate Fellowship and a grant to the University of British Columbia from the British Columbia Ministry of Health.

\section{Competing interests None.}

Ethics approval The study protocol was approved by the Clinical Research Ethics Board of the University of British Columbia (number H11-00303).

Provenance and peer review Not commissioned; externally peer reviewed.

Data sharing statement Patient level data are available from Population Data BC (https://www.popdata.bc.ca/). Statistical code is available from the corresponding author.

Open Access This is an Open Access article distributed in accordance with the Creative Commons Attribution Non Commercial (CC BY-NC 4.0) license, which permits others to distribute, remix, adapt, build upon this work noncommercially, and license their derivative works on different terms, provided the original work is properly cited and the use is non-commercial. See: http:// creativecommons.org/licenses/by-nc/4.0/

\section{REFERENCES}

1. Cush JJ. Biological drug use: US perspectives on indications and monitoring. Ann Rheum Dis 2005;64(Suppl 4):iv18-23.

2. Curtis JR, Chen L, Harrold LR, et al. Physician preference motivates the use of anti-tumor necrosis factor therapy independent of clinical disease activity. Arthritis Care Res (Hoboken) 2010;62:101-7.

3. Schneeweiss S, Glynn RJ, Avorn J, et al. A Medicare database review found that physician preferences increasingly outweighed patient characteristics as determinants of first-time prescriptions for COX-2 inhibitors. J Clin Epidemiol 2005;58:98-102.

4. Solomon DH, Schneeweiss S, Glynn RJ, et al. Determinants of selective cyclooxygenase-2 inhibitor prescribing: are patient or physician characteristics more important? Am J Med 2003;115:715-20.

5. Zhang J, Shan Y, Reed G, et al. Thresholds in disease activity for switching biologics in rheumatoid arthritis patients: experience from a large US cohort. Arthritis Care Res 2011;63:1672-9.

6. Sokka T, Pincus T. Eligibility of patients in routine care for major clinical trials of anti-tumor necrosis factor alpha agents in rheumatoid arthritis. Arthritis Rheum 2003;48:313-18.

7. Zink A, Strangfeld A, Schneider M, et al. Effectiveness of tumor necrosis factor inhibitors in rheumatoid arthritis in an observational cohort study: comparison of patients according to their eligibility for major randomized clinical trials. Arthritis Rheum 2006;54:3399-407. 
8. Kievit W, Fransen J, Oerlemans AJ, et al. The efficacy of anti-TNF in rheumatoid arthritis, a comparison between randomised controlled trials and clinical practice. Ann Rheum Dis 2007;66:1473-8.

9. Berthelot JM, Benoist-Gerard S, le Goff B, et al. Outcome and safety of TNF alpha antagonist therapy in 475 consecutive outpatients (with rheumatoid arthritis or spondyloarthropathies) treated by a single physician according to their eligibility for clinical trials. Joint Bone Spine 2010;77:564-9.

10. Lacaille D, Anis AH, Guh DP, et al. Gaps in care for rheumatoid arthritis: a population study. Arthritis Rheum 2005;53:241-8.

11. Lacaille D, Guh DP, Abrahamowicz M, et al. Use of nonbiologic disease-modifying antirheumatic drugs and risk of infection in patients with rheumatoid arthritis. Arthritis Rheum 2008;59:1074-81.

12. Li X, Guh D, Lacaille D, et al. The impact of cost sharing of prescription drug expenditures on health care utilization by the elderly: own- and cross-price elasticities. Health Policy 2007;82:340-7.

13. Your level of Fair PharmaCare coverage in Fair PharmaCare. http:// www.health.gov.bc.ca/pharmacare/plani/planiindex.html\#7 (accessed 5 Mar 2011).

14. Charlson ME, Pompei $\mathrm{P}$, Ales $\mathrm{KL}$, et al. A new method of classifying prognostic comorbidity in longitudinal studies: development and validation. J Chronic Dis 1987;40:373-83.

15. Quan H, Sundararajan V, Halfon $P$, et al. Coding algorithms for defining comorbidities in ICD-9-CM and ICD-10 administrative data. Med Care 2005;43:1130-9.

16. Widdifield J, Bernatsky S, Paterson JM, et al. Accuracy of Canadian health administrative databases in identifying patients with rheumatoid arthritis: a validation study using the medical records of rheumatologists. Arthritis Care Res (Hoboken) 2013;65:1582-91.

17. Bensen $B$, Atkins $C$, Sheriff $M$, et al. Profile of patients with rheumatoid arthritis treated with infliximab in Canada-trends toward less DMARD use prior to a biologic, earlier use of infliximab and differences in baseline disease [abstract]. J Rheumatol 2011;38:1155.

18. Buchanan J, Rahman MU, Doyle MK, et al. Historical trends of patient characteristics in anti-TNF clinical trials for rheumatoid arthritis: an analysis of the literature over the past 16 years [abstract]. Arthritis Rheum 2009;60(Supple 10):1012.

19. Hyrich KL, Watson KD, Lunt M, et al.; on behalf of the British Society for Rheumatology Biologics Register (BSRBR). Changes in disease characteristics and response rates among patients in the United Kingdom starting anti-tumour necrosis factor therapy for rheumatoid arthritis between 2001 and 2008. Rheumatology (Oxford) 2011;50:117-23.

20. Lee SJ, Chang $\mathrm{H}$, Yazici $\mathrm{Y}$, et al. Utilization trends of tumor necrosis factor inhibitors among patients with rheumatoid arthritis in a United States observational cohort study. J Rheumatol 2009;36:1611-17.

21. Lee EW, Wei LJ, Amato DA. Cox-type regression analysis for large numbers of small groups of correlated failure time observations. In: Klein JP, Goel PK, eds. Survival analysis: state of the art. NATO ASI series. Series E, Applied sciences edition. Boston, MA: Kluwer Academic Publishers, 1992:237-47.

22. Statistical analysis of clustered data using $S A S \circledR$ system (NESUG 2006). http://www.nesug.org/proceedings/nesug06/an/da01.pdf (accessed 12 Nov 2010).

23. Analysis of Survival Data with Clustered Events (paper 237-2009). http://support.sas.com/resources/papers/proceedings09/237-2009. pdf (accessed 12 Nov 2010)

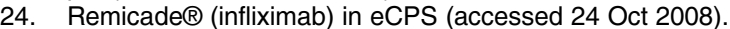

25. Enbrel® (Etanercept) in eCPS (accessed 3 Jun 2009).

26. Special Authority request; A batacept/adalimumab/certolozumab/ etanercept/golimumab/infliximab rheumatoid arthritis (initial or switch). https://www.health.gov.bc.ca/exforms/pharmacare/5345fil. pdf (accessed 10 May 2012).

27. Mark TL, Swait J. Using stated preference and revealed preference modeling to evaluate prescribing decisions. Health Econ 2004;13:563-73.
28. Wolfe F. The epidemiology of drug treatment failure in rheumatoid arthritis. Baillieres Clin Rheumatol 1995;9:619-32.

29. Hyrich $\mathrm{KL}$, Lunt M, Watson KD, et al. Outcomes after switching from one anti-tumor necrosis factor alpha agent to a second anti-tumor necrosis factor alpha agent in patients with rheumatoid arthritis: results from a Large UK National Cohort Study. Arthritis Rheum 2007;56:13-20.

30. Carmona L, Gomez-Reino JJ; on behalf of the BIOBADASER Group. Survival of TNF antagonists in spondylarthritis is better than in rheumatoid arthritis. Data from the Spanish registry BIOBADASER. Arthritis Res Ther 2006;8:R72.

31. Hetland ML, Christensen IJ, Tarp U, et al. Direct comparison of treatment responses, remission rates, and drug adherence in patients with rheumatoid arthritis treated with adalimumab, etanercept, or infliximab: results from eight years of surveillance of clinical practice in the nationwide Danish DANBIO registry. Arthritis Rheum 2010;62:22-32.

32. Fernandez-Nebro A, Irigoyen MV, Urena I, et al. Effectiveness, predictive response factors, and safety of anti-tumor necrosis factor (TNF) therapies in anti-TNF-naive rheumatoid arthritis. J Rheumatol 2007;34:2334-42.

33. Kievit W, Fransen J, Adang EMM, et al. Long-term effectiveness and safety of TNF-blocking agents in daily clinical practice: results from the Dutch Rheumatoid Arthritis Monitoring Register. Rheumatology (Oxford) 2011;50:196-203.

34. Filippini M, Bazzani C, Favalli EG, et al. Efficacy and safety of anti-tumour necrosis factor in elderly patients with rheumatoid arthritis: an observational study. Clin Rev Allergy Immunol 2010;38:90-6.

35. Baser O, Wang L, Xie L, et al. Deriving doctors' prescribing patterns from health care claims: an instrumental variable analysis [abstract]. Value Health 2010;13:A425.

36. Kamal KM, Madhavan SS, Hornsby JA, et al. Use of tumor necrosis factor inhibitors in rheumatoid arthritis: a national survey of practicing United States rheumatologists. Joint Bone Spine 2006;73:718-24.

37. Festinger L. A theory of cognitive dissonance. Evanston, IL: Row, Peterson, 1957

38. Visser PS, Cooper J. Dissonance and attitude change: a matter of measurement, in chapter 10: attitude change. In: Hogg MA Cooper J, eds. The Sage handbook of social psychology. Thousand Oaks, CA: Sage, 2003:220.

39. Yazici Y, Simsek I. Treatment options for rheumatoid arthritis beyond TNF- $\alpha$ inhibitors. Expert Rev Clin Pharmacol 2010; 3:663-6.

40. Dudler J, Moller B, Michel BA, et al. Biologics in rheumatoid arthritis (RA)-recommendations for Swiss practice. Swiss Med Wkly 2011;141:w13189.

41. Lu TY, Hill C. Managing patients taking tumour necrosis factor inhibitors. Aust Prescr 2006;29:67-70.

42. Gall EP, Caperton EM, McComb JE, et al. Clinical comparison of ibuprofen, fenoprofen calcium, naproxen and tolmetin sodium in rheumatoid arthritis. J Rheumatol 1982;9:402-7.

43. Wasner C, Britton MC, Kraines RG, et al. Nonsteroidal anti-inflammatory agents in rheumatoid arthritis and ankylosing spondylitis. JAMA 1981;246:2168-72.

44. Chilton F, Collett RA. Treatment choices, preferences and decision-making by patients with rheumatoid arthritis. Musculoskeletal Care 2008;6:1-14.

45. Rheumatoid Arthritis Patient Preference Survey (RAPPS) (Presentation number: 965). http://acr.confex.com/acr/2006/ webprogram/Paper5515.html (accessed 5 Dec 2011).

46. Spurling GK, Mansfield PR, Montgomery BD, et al. Information from pharmaceutical companies and the quality, quantity, and cost of physicians' prescribing: a systematic review. PLoS Med 2010;7: e1000352. 